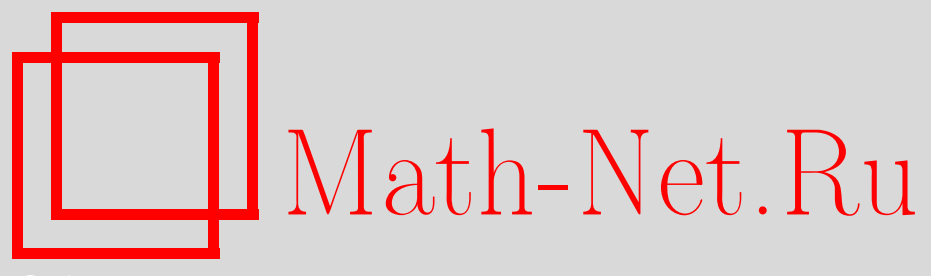

В. М. Бухштабер, А. А. Гайфуллин, Представления $m$ значных групп на триангуляциях многообразий, $У M H$, 2006, том 61, выпуск 3, 171-172

DOI: https://doi.org/10.4213/rm1751

Использование Общероссийского математического портала Math-Net.Ru подразумевает, что вы прочитали и согласны с пользовательским соглашением http: //www . mathnet.ru/rus/agreement

Параметры загрузки:

IP : 3.85 .7 .115

26 апреля 2023 г., 15:15:36

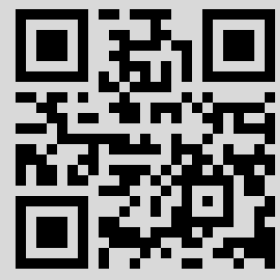




\section{Представления $m$-значных групп на триангуляциях многообразий}

\section{В. М. Бухштабер, А. А. Гайфуллин}

Предлагается подход к характеризации триангуляций многообразий средствами теории $m$-значных групп.

Для произвольного множества $X$ через $(X)^{m}$ мы обозначим его $m$-ю симметрическую степень. Говорят, что на множестве $X$ задана структура $m$-значной группь, если заданы $m$-значная операция умножения $\mu: X \times X \rightarrow(X)^{m}, \mu(x, y)=x * y$, единица $e \in X$ и операция взятия обратного элемента inv : $X \rightarrow X$, удовлетворяющие естественным обобщениям аксиом ассоциативной группы (см. [1]). Для любых группы $G$ и ее конечной подгруппы $H$ из $m$ элементов на множестве двойных смежных классов $H \backslash G / H$ существует структура бикосетной $m$-значной группы с умножением $\left(H h_{1} H\right) *\left(H h_{2} H\right)=\left[H h_{1} h h_{2} H, h \in H\right]$.

Действием $m$-значной группы $X$ на множестве $S$ называется отображение $X \times S \rightarrow$ $(S)^{m},(x, s) \mapsto x \circ s$, такое, что для любых $x_{1}, x_{2} \in X, s \in S$ наборы $\left(x_{1} * x_{2}\right) \circ s$ и $x_{1} \circ\left(x_{2} \circ s\right)$ из $m^{2}$ элементов совпадают, $e \circ s=[s, \ldots, s]$.

Отображение $T: S \rightarrow(S)^{m}$ называется $m$-значной динамикой на множестве $S$. Говорят, что $m$-значная динамика $T$ интегрируема при помощи $m$-значной группы $X$ с одной образующей $a$, если существует действие группы $X$ на множестве $S$ такое, что $T s=a \circ s$ для любого $s \in S$.

Для любого $k$ структура $m$-значной группы на множестве $X$ определяет на $X$ структуру $k m$-значной группы $k X$, называемой диагоналъю исходной группы. Аналогично определяется диагональ $m$-значной динамики (детали см. в [1]).

Пусть $K$ - симплициальный или симплициально клеточный комплекс (см. [2]), $K(n-2)$ - дополнение к его $(n-2)$-мерному остову. Комплекс $K$ называется $n$-мерным псевдомногообразием, если каждый его симплекс содержится в некотором симплексе размерности $n$, причем каждый $(n-1)$-мерный симплекс содержится ровно в двух симплексах размерности $n$, и пространство $K(n-2)$ связно.

Для каждого $n$-мерного псевдомногообразия $K$ определена $(n+1)$-значная динамика $T$ на множестве $S$ его $n$-мерных симплексов, которая каждому симплексу $\sigma$ сопоставляет набор всех $n$-мерных симплексов, не совпадающих с $\sigma$ и имеющих с $\sigma$ общую $(n-1)$-мерную грань. В случае симплициально клеточного комплекса симплекс, имеющий с $\sigma$ несколько общих $(n-1)$-мерных граней, входит в набор $T \sigma$ с соответствующей кратностью. Обозначим через $K^{\prime}$ барицентрическое подразделение комплекса $K$ и через $S^{\prime}$ множество $n$-мерных симплексов комплекса $K^{\prime}$.

Теорема 1. Для любого псевдомногообразия $K$ динамика $n ! T$ интегрируема при помощи некоторой бикосетной $(n+1)$ !-значной группы $X=H \backslash G / H$ с одной образующей. При этом в качестве группь $G$ может быть выбрана некоторал подгруппа группы перестановок $\Sigma_{S^{\prime}}$ множества $S^{\prime}$, а подгруппа $H$ изоморфна группе $\Sigma_{n+1}$.

ДоказАтельство. Дадим явную конструкцию $(n+1)$ !-значной группы $X$. Рассмотрим симплекс $\Delta^{n}$ с множеством вершин $\{0,1, \ldots, n\}$ и каноническое симплициальное отображение $\pi: K^{\prime} \rightarrow \Delta^{n}$, переводящее вершину $\alpha \in K^{\prime}$ в вершину $\pi(\alpha)=i \in$ $\Delta^{n}$, где $i$ - размерность симплекса $\sigma \in K$, барицентром которого является $\alpha$. Ясно, что ограничение отображения $\pi$ на любой $n$-мерный симплекс из $K^{\prime}$ является гомеоморфизмом. Скажем, что вершина $\alpha \in K^{\prime}$ имеет тип $i$, если $\pi(\alpha)=i$, и $(n-1)$-мерный симплекс $\rho \in K^{\prime}$ имеет тип $i$, если $i$ не является вершиной симплекса $\pi(\rho)$.

Для каждого целого числа $i, 0 \leqslant i \leqslant n$, и любого $n$-мерного симплекса $\tau$ из $K^{\prime}$ обозначим через $\Phi_{i}(\tau)$ (единственный) $n$-мерный симплекс комплекса $K^{\prime}$, имеющий с $\tau$ общую $(n-1)$-мерную грань типа $i$. Таким образом, мы получаем корректно 
определенные перестановки $\Phi_{i} \in \Sigma_{S^{\prime}}, 0 \leqslant i \leqslant n$. Возьмем в качестве $G$ подгруппу группы $\Sigma_{S^{\prime}}$, порожденную этими перестановками, и в качестве $H$ - подгруппу, порожденную перестановками $\Phi_{0}, \Phi_{1}, \ldots, \Phi_{n-1}$.

ЛЕмма 1. Перестановки $\Phi_{i}$ удовлетворяют следующим соотношениям:

$$
\Phi_{i}^{2}=1 ; \quad \Phi_{i} \Phi_{j}=\Phi_{j} \Phi_{i}, \quad|i-j|>1 ; \quad \Phi_{i} \Phi_{i+1} \Phi_{i}=\Phi_{i+1} \Phi_{i} \Phi_{i+1}, \quad i<n-1 .
$$

Группа $H$ изоморфна $\Sigma_{n+1}$.

Рассмотрим бикосетную $(n+1)$ !-значную группу $X=H \backslash G / H$ с одной образующей $a=H \Phi_{n} H$. Группа $G$ действует на множестве $S^{\prime}$ так, что $S^{\prime} / H=S$. Следовательно, имеет место действие $(n+1)$ !-значной группы $X$ на множестве $S$. Несложно проверить, что это действие интегрирует $(n+1)$ !-значную динамику $n ! T$.

Если $K=\partial \Delta^{n+1}$, то $(n+1)$ !-значная группа $X$ является диагональю некоторой двухэлементной $(n+1)$-значной группы, которая в [1] обозначается через $X\left(\mathbb{Z}_{2}, 1, n\right)$. Следовательно, в этом случае динамика $T$ интегрируема.

В общем случае предъявленная $(n+1)$ !-значная группа $X$ не является диагональю какой-либо $(n+1)$-значной группы. Вопрос о характеризации $n$-мерных псевдомногообразий с динамикой $T$, интегрируемой при помощи $(n+1)$-значной группы, остается открытым.

Будем говорить, что $n$-мерная триангуляция $K$ является симметричной, если группа Aut $K$ ее симплициальных автоморфизмов действует транзитивно на множестве $n$-мерных симплексов ее барицентрического подразделения $K^{\prime}$. Симплициальное отображение $\widehat{K} \rightarrow K$ назовем почти накрытием, если индуцированное отображение $\widehat{K}(n-2) \rightarrow K(n-2)$ является накрытием.

Теорема 2. Имеет место явная конструкиия, сопоставляющая каждому п-мерному псевдомногообразию $K$ минимальное симметричное псевдомногообразие $\widehat{K} u$ почти накрытие $\widehat{K} \rightarrow K$ такие, что если $\widetilde{K} \rightarrow K$ - почти накрытие $и \widetilde{K}-$ симметричное псевдомногообразие, то соответствующее $\widetilde{K}$ накрытие $\widetilde{K}(n-2) \rightarrow K(n-2)$ пропускается через $\widehat{K}(n-2)$. Группа $G$ из теоремы 1 изоморфна группе Aut $\widehat{K}$.

Динамика $T$ на множестве $n$-мерных симплексов $n$-мерного псевдомногообразия может быть реализована в виде канонической $(n+1)$-значной динамики на множестве вершин соответствующего однородного графа степени $n+1$. Оказывается, что описанные выше методы применимы в более общем случае.

Теорема 3. Пусть $T$ - каноническая $(n+1)$-значная динамика на множестве вершин произвольного однородного графа степени $n+1$. Пусть $G_{n}-$ группа с образуюощии $\Phi_{0}, \Phi_{1}, \ldots, \Phi_{n}$ и соотношениями из леммы $1, H_{n}$ - ее подгруппа, порожденная образующими $\Phi_{0}, \Phi_{1}, \ldots, \Phi_{n-1}$. Тогда динамика $n ! T$ интегрируема при помощи $(n+1)$-значной группы $X_{n}=H_{n} \backslash G_{n} / H_{n}$.

\section{Список литературы}

[1] V. M. Buchstaber, " $n$-valued groups: theory and applications", Moscow Math. J., 6:2 (2006). [2] В. М. Бухштабер, Т. Е. Панов, Тр. МИАН, 247 (2004), 41-58.

\section{B. М. Бухштабер (V. M. Buchstaber)}

Математический институт им. В. А. Стеклова РАН

E-mail: buchstab@mi.ras.ru
Представлено С. П. Новиковым Принято редколлегией 02.05.2006

\section{А. А. Гайфуллин (А. A. Gařfullin)}

Московский государственный университет им. М. В. Ломоносова

E-mail: gaifull@mccme.ru 